\title{
Baduangong Exercise Provides New Insights into Neck Type of Cervical Spondylosis
}

\author{
Shudong Chen ${ }^{1, a}$, Guoyi Su², HongZhi Xu², Binde Zhao², \\ Yu Hou ${ }^{2}$, Dingkun Lin ${ }^{1,2, b *}$ \\ ${ }^{1}$ Second School of Clinic Medicine, Guangzhou University of Chinese Medicine, No. 12 Jichang Road, \\ Baiyun District, Guangzhou, 510405, China \\ ${ }^{2}$ Guangdong Provincial Hospital of Traditional Chinese Medicine, Guangdong Provincial Academy of \\ Chinese Medical Sciences, No.111 Dade Road, Yuexiu District, Guangzhou, 510120, China \\ achenshudong_med@163.com, bomorrow2008@126.com
}

\section{Keywords: Traditional Chinese Medicine; Exercise; Cervical Spondylosis}

\begin{abstract}
Objective: To evaluate the effects of manual therapy combine with Baduangong exercise to treat neck-type cervical spondylosis. Methods: Patients were randomized to control group (cervical manipulation) and experimental group (combining cervical manipulation with Baduangong exercise). The two groups received manipulation of the cervical spine alone every other day for 2 weeks, and the experimental group performed Baduangong also. VAS scale and NDI were all completed. Results: There were 21 patients, 12 in control group and 9 in experimental group. The mean age of the patients is $39.1 \pm 5.2$ years. Mean VAS score and NDI of the control group is $3.31 \pm 0.99$ and $28.67 \pm 7.88$, while $3.74 \pm 0.92,27.11 \pm 2.93$ in experimental group, respectively. After treatment, two groups reduce pain significantly, and the experimental group showed a quick improvement. 4 patients $(33.3 \%)$ relapse in control group and 1 patient $(11.1 \%)$ in experimental group. Significant differences were found in between pre and pro-treat $(\mathrm{P}<0.05)$, however, there was no statistics difference between two groups ( $\mathrm{P}>0.05)$. Conclusion: It is suggested that manual therapy can relieve neck pain, moreover, combine with Baduangong exercise, it is more beneficial to relieve neck and reduce the recurrence rate.
\end{abstract}

\section{Introduction}

Neck type of cervical spondylosis is a common disease and frequently encountered in outpatient department. With social progress and quick development of electronics, it shows an increased prevalence. Neck pain is regarded as an early stage of other various types of cervical spondylosis. Many people suffered neck pain ever. If the pain progress and turns to cervical spondylotic radiculopathy or cervical spondylotic myelopathy, patients' health were affected seriously and someone had to undergo a surgery.

NSAIDs, skeletal muscular relaxants, opioids, steroids, and others can control neck pain well. However, some people might suffer side effects. Although the cause of cervical spondylosis may be related to cervical degeneration, we all know that the tissue play an important role in the neck pain. Manipulation, one of the Traditional Chinese Medicine therapies, shows good curative effect on muscle relaxation [1,2]. But today, exercise therapies, for example, Tai Chi, Pilates, are more attractive to those patients. It can relieve neck pain as well and reduce the recurrence rate, but there are no more evidences to support it. Thus, we carried out a study and use Baduangong, reformed from some traditional Chinese health-promoting exercises, to evaluate the clinical outcomes of exercise therapy to treat neck type of cervical spondylosis. 


\section{Methods}

Participants. All the patients came from the out-patient department at the Department of Orthopedics, Guangdong Provincial Hospital of Chinese Medicine. All the participated patients signed an informed consent form accepted by the Ethical committee of Guangdong Provincial Hospital of Chinese Medicine. The diagnosis of neck type of cervical spondylosis is from the Diagnosis and treatment of cervical spondylosis and rehabilitation guide (2010), Chinese Association of Rehabilitation Medicine. The inclusion criteria were following: age between 30 to 50, stiff neck, neck or shoulder pain, cervical and upper thoracic regions movement limitation. People who take NSAIDs or other drugs were excluded.

Intervention. Patients were randomized to cervical manipulation as control group and combining cervical manipulation with exercise as experimental group. The two groups received manipulation of the cervical spine alone every other day for 2 weeks. In addition, the experimental group performed Baduangong, which involves eight actions (Fig. 1). It has standardized by Pro.Dinkun Lin and made into DVD. In this study, Baduangong was led by team members, all through the strict training. Patients were required to attend 20 min sessions 3 times a week to learn and perform this exercise and were also asked to practice it every day.

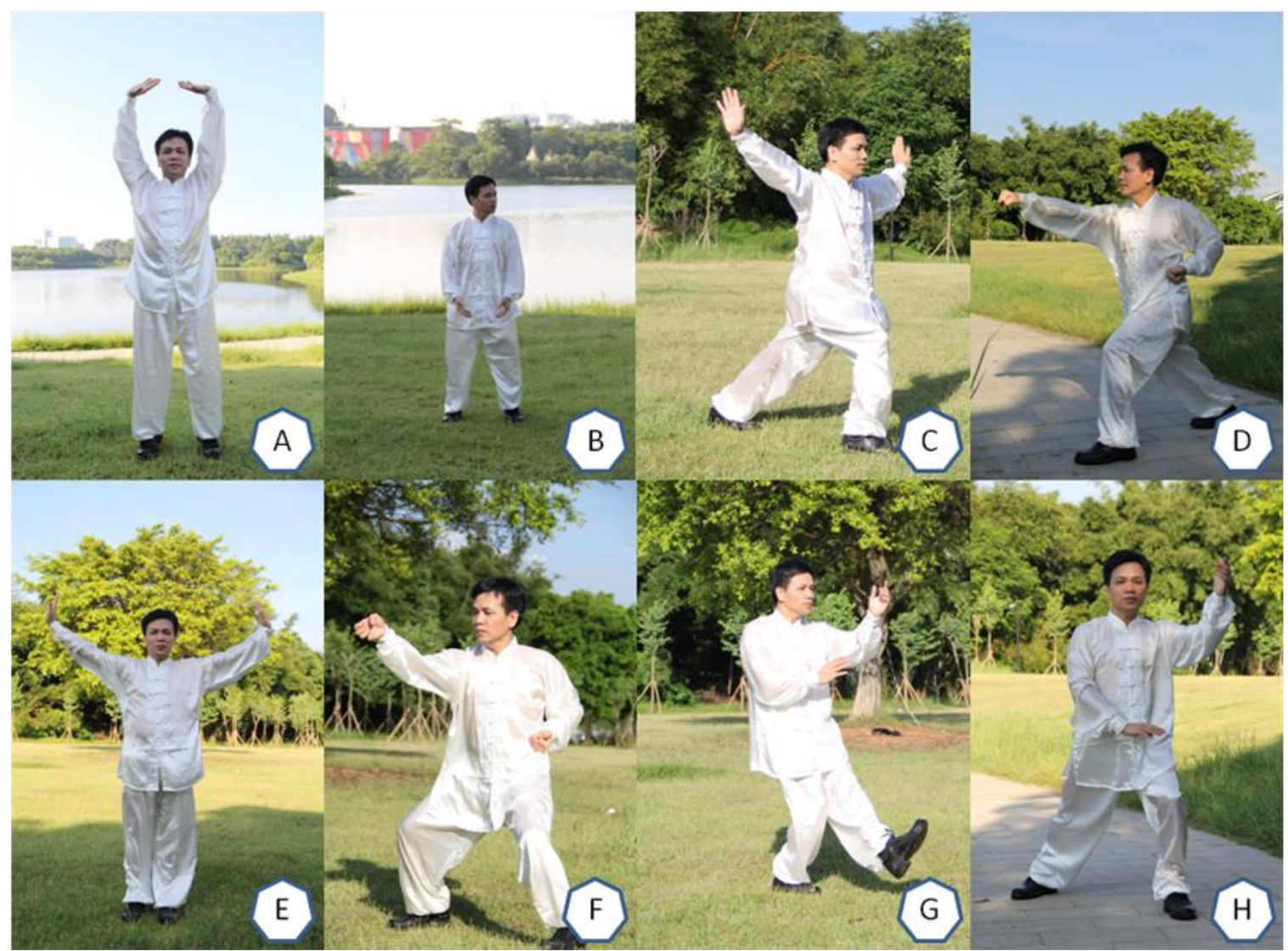

Figure. 1 The Standardized Baduangong Exercise by Pro. Dinkun Lin

A: Prop up the sky with both hands to regulate the triple warmer; B: Put hands on both sides and strain muscles of limbs; C: Step forwards and lunges with both hands extending; D: Move forward and Soopyong Jirugi; E: Stand up straight and like bird spreads the wings; F: Grabbing hand with the"horse stance" posture; G: Squat and Leg Kick to move along with block; H: Horse stance cloud hands to balance Yin-Yang 


\section{Outcome Measures}

Visual analogue scale (VAS), and Neck disability index (NDI) [3] were evaluated before and during the follow-up. Patients were asked to visit the outpatient clinic to fill the scales at two weeks, 1 month and 3 months. Telephone interviews were demanded if the patient can't visit the clinic.

\section{Statistical Analysis}

Data were entered and analyzed in SPSS 18.0. And for the count data, Chi-square test was applied. Results were considered of statistical significance when $\mathrm{p}<0.05$.

\section{Results}

There were 21 patients, 12 in control group and 9 in experimental group from May, 2014 to November, 2014 in our hospital. The age of the patients ranged from 33-48 (39.1 55.2$)$ years. The baseline data showed in Table 1.

There was no patient lost to follow-up and no one progress to other type of cervical spondylosis. There were 4 patients $(33.3 \%)$ relapse in control group and 1 patient $(11.1 \%)$ in experimental group. The VAS score and NDI score were showed in Table 2. The scores of VAS and NDI after treatment reduced significantly compare to pre-treat, and it continued to lower during follow up. Significant differences were found in between pre and pro-treat $(\mathrm{P}<0.05)$, however, there was no statistics difference between two groups $(\mathrm{P}>0.05)$.

Table 1 Characteristic of two groups

\begin{tabular}{ccccc}
\hline Group & Cases & Male & Female & Age \\
\hline Control & 12 & 7 & 5 & $38.8 \pm 5.3$ \\
Experimental & 9 & 4 & 5 & $39.6 \pm 5.4$ \\
Total & 21 & 11 & 10 & $39.1 \pm 5.2$ \\
\hline
\end{tabular}

Table 2 Comparison of VAS and NDI score in two groups

\begin{tabular}{lllllllll}
\hline Group & \multicolumn{2}{c}{ Pre-treat } & \multicolumn{2}{c}{ Two weeks } & \multicolumn{2}{c}{1 months } & \multicolumn{2}{c}{3 months } \\
\hline & VAS & NDI & VAS & NDI & VAS & NDI & VAS & NDI \\
\hline Control & $3.31 \pm 0.99$ & $28.67 \pm 7.88$ & $1.46 \pm 0.66$ & $6.17 \pm 2.41$ & $1.23 \pm 0.56$ & $8.25 \pm 1.82$ & $0.58 \pm 0.51$ & $4.83 \pm 1.34$ \\
Experimental & $3.74 \pm 0.92$ & $27.11 \pm 2.93$ & $1.50 \pm 0.43$ & $6.78 \pm 3.15$ & $1.30 \pm 0.46$ & $7.63 \pm 2.64$ & $0.44 \pm 0.46$ & $2.89 \pm 1.62$ \\
\hline
\end{tabular}

\section{Disscusion}

According to the Neck pain-Clinical Practice Guidelines [4] from the Orthopaedic Section of the American Physical Therapy Association recommendation, cervical manipulation and mobilization procedures can reduce neck pain with strong evidence. However, combining with exercise is more beneficial to relieve neck pain than manipulation and mobilization alone. Traditional Chinese exercises, like Tai Chi [5, 6], Qigong [5], are growing evidence base to support the beneficial effects on many disease. Baduangong, reformed from Tai Chi, Qigong and Gongfu, provide a more appropriate choice for young people who suffer chronic pain because of its high quality of training. Baduangong is simple, practicable and low physically demanding. It consists of eight actions, and includes flexibility, strengthening, endurance and coordination exercises. In this study, patients reduced pain quickly after two weeks treatment, but in experimental group was more significantly. Furthermore, it was found to improve the neck movement and quality of life according to the NDI score. 
Weakness of the cervical and upper thoracic muscle could result in significant axial pain. Muscle training should be given attention. Manual therapy can relieve muscle fatigue and tightness. Allison GT et al [7] presented that manipulation for cervico-brachial pain syndrome can get a significantly lower pain score. Ylinen et al $[8,9]$ found that neck muscle training could increase strength of muscle and relieve the pain and enhance the activities of the patients' daily life. Additionally, studies indicated that combined program of strengthening and endurance exercises with manual therapy got a greater result in range of motion, and long-term pain relieve than manipulation only $[10,11]$.Our results are similar to previous studies. Baduangong contribute to the physical function, for example, the movement of the extremities, especially the muscles around the shoulders. Compared to the control group, patients who performed Baduangong may improve healthy and have positive effects on functional balance as well.

Manual therapy is a good method for neck pain. And Baduangong can benefit to the patients in our study, but further studies should be composed of larger cases and have a long-term follow up. What's more, exercise is a physical intervention, it might not be as easy to ensure all participants can insist to practice, so further study is also needed to evaluate other indexes, such as patient compliance, amount of exercise and so on.

\section{Acknowledgements}

This study was funded by and in part of Construction model of community health management for Middle-aged patients with cervical spondylosis (Guangdong province science and technology plan projects, No. 2012KT1421) to Lin.

\section{References}

[1] Q. Ding, M. Yan, J. Zhou, L. Yang, J. Guo, J. Wang, Z. Shi, Y. Wang and H. Zhao: Clinical effects of innovative tuina manipulations on treating cervical spondylosis of vertebral artery type and changes in cerebral blood flow, J Tradit Chin Med. Vol. 32(2012), p.388-392.

[2] L.J. Kong, M. Fang, H.S. Zhan, W.A. Yuan, J.H. Pu, Y.W. Cheng and B. Chen: Tuina-focused integrative chinese medical therapies for inpatients with low back pain: a systematic review and meta-analysis, Evid Based Complement Alternat Med. Vol. 2012(2012), p.578305.

[3] H. Vernon, S. Mior: The Neck Disability Index: a study of reliability and validity, J Manipulative Physiol Ther. Vol. 14(1991), p.409-415.

[4] J.D. Childs, J.A. Cleland, J.M. Elliott, D.S. Teyhen, R.S. Wainner, J.M. Whitman, B.J. Sopky, J.J. Godges and T.W. Flynn: Neck Pain: Clinical Practice Guidelines Linked to the International Classification of Functioning, Disability, and Health From the Orthopaedic Section of the American Physical Therapy Association,J Orthop Sports Phys Ther. Vol. 38 (2008), p.A1-A34.

[5] Y. Yang, J. Verkuilen, K.S. Rosengren, R.A. Mariani, M. Reed, S.A. Grubisich and J.A. Woods: Effects of a Taiji and Qigong intervention on the antibody response to influenza vaccine in older adults, Am. J. Chin. Med, Vol. 35(2007), p.597-607.

[6] P.J. Klein, W.D. Adams: Comprehensive therapeutic benefits of Taiji: a critical review, Am J Phys Med Rehabil, Vol. 83(2004), p.735-745.

[7] G.T. Allison, B.M. Nagy and T. Hall: A randomized clinical trial of manual therapy for cervico-brachial pain syndrome - a pilot study, Man Ther, Vol. 7(2002), p.95-102.

[8] J.J. Ylinen, E.P. Takala and H. Kautiainen: Effect of longterm neck muscle training on pressure pain threshold:a randomized controlled trial, Eur J Pain, Vol. 9(2005), p.673.

[9] J.J. Ylinen, E.P. Takala and M.J. Nyknen: Effects of twelve month strength training subsequent to twelve month stretching exercise in treatment of chronic neck pain, J Strength Cond Res, Vol. 20(2006), p.304. 
[10] R. Evans, G. Bronfort, B. Nelson and C.H. Goldsmith: Two-year follow-up of a randomized clinical trial of spinal manipulation and two types of exercise for patients with chronic neck pain, Spine, Vol. 27(2002), p.2383-2389.

[11] T.T. Chiu, T.H. Lam and A.J. Hedley: A randomized controlled trial on the efficacy of exercise for patients with chronic neck pain, Spine, Vol. 30(2005), p.E1-7. 